\title{
Diplomaten zwischen Sozialisation und Kalkulation: Bestimmungsfaktoren des Berufserfolgs im Auswärtigen Dienst
}

\author{
Nicole Koenig • Gerald Schneider
}

Zusammenfassung: Der Artikel untersucht, welche Faktoren den Berufserfolg von Karrierediplomaten im deutschen Auswärtigen Dienst bestimmen. Ausgehend vom Sozialisations- und Rational-Choice-Ansatz konstruieren wir zwei Modelle, deren Erklärungskraft wir anschließend anhand von Daten aus vier Berufseinstiegskohorten (Attaché-Lehrgänge 1970-1973) evaluieren. Die statistische Analyse einer systematischen Befragung zeigt, dass der Berufserfolg stärker durch Aspekte des Karrierekalküls, wie etwa Netzwerkarbeit und Konformismus, als durch gruppenspezifische Charakteristika, wie den Amtskindbonus oder die Parteimitgliedschaft, beeinflusst wird. Auch wenn die unterschiedlichen Erklärungsmodelle schwer voneinander trennbar sind, erweisen sich die rationalistischen Faktoren für den monetären Berufserfolg erklärungsmächtiger als jene Faktoren, die wir dem Sozialisationsmodell zugeordnet haben.

Schlüsselwörter: Außenpolitikanalyse $\cdot$ Rational Choice $\cdot$ Konstruktivismus $\cdot$ Diplomatie $\cdot$ Eliteninterviews

\section{Diplomats between Socialization and Calculation: Determinants of Professional Success in the German Foreign Office}

Abstract: This article examines the determining factors of career success in the German Foreign
Service. Based on the socialization and the rational choice approach, we design two models from
which empirical relevance is evaluated through data from four career entry cohorts (attache trai-
ning courses 1970-1973). The statistical analysis of a systematic survey shows that facets of

Dipl.-Verw.wissenschaftlerin N. Koenig, M.A.

Lehrstuhl für Internationale Politik, Universität Konstanz, Universitätsstr. 10

78457 Konstanz, Deutschland

E-Mail: Nicole.Koenig@coleurope.eu

Prof. Dr. G. Schneider (价)

Fachbereich Politik- und Verwaltungswissenschaft, Lehrstuhl fur Internationale Politik,

Universităt Konstanz, Universitătsstr. 10,

78457 Konstanz, Deutschland

E-Mail: Gerald.Schneider@uni-konstanz.de

Konstanzer Online-Publikations-System (KOPS)

URN: http://nbn-resolving.de/urn:nbn:de:bsz:352-opus-121196

URL: http://kops.ub.uni-konstanz.de/volltexte/2010/12119 
career calculation such as networking or conformity more strongly influence the professional achievements of a diplomat than that of group-specific features such as family background or party membership. Even though we cannot strictly separate the models from each other, it becomes evident that the rationalist explanation predicts career success better than the factors attributed from the socialization model.

Keywords: Foreign policy analysis - Rational choice Constructivism - Diplomacy Elite interviews

\section{Einleitung}

Wer besetzt die Spitzenpositionen in der deutschen Außenpolitik und warum? Vordergründig lautet die Antwort: die durch Wahlen legitimierte politische Führung - also der Bundeskanzler, der Außenminister und das Kabinett sowie der Bundespräsident, der für die völkerrechtliche Vertretung der Bundesrepublik zuständig ist. Wenn man jedoch der These der „verwalteten Außenpolitik“ (Haftendorn 1978) Glauben schenkt, sind die eigentlichen außenpolitischen Akteure die Beamten der Ministerialbürokratie und ihre Gegenparts in internationalen Stäben und Sekretariaten - mit anderen Worten: die Diplomaten ${ }^{1}$. Sie bereiten die Entscheidungen der politischen Führung vor, sind maßgeblich an der Gestaltung der Außenpolitik beteiligt und im In- und Ausland für ihre Durchführung zuständig.

Doch wer ist diese einflussreiche Elite an den Schnittstellen der deutschen Außenpolitik? Wo kommt sie her, und wie verläuft ihr beruflicher Werdegang? Während es mittlerweile eine Vielzahl von Elitenuntersuchungen gibt (z. B. Enke 1974; HoffmannLange 1990a, 1990b; Wildenmann et al. 1982; Bürklin u. Rebenstorf 1997), bewahrt das diplomatische Corps weiterhin berufsspezifische Anonymität. Man sollte annehmen, dass die wichtige Stellung der Diplomaten in der Außenpolitik, ihr hohes soziales Prestige sowie die Mythen, die sie umgeben, zu wissenschaftlichen Untersuchungen anregen. Der Forschung in diesem Bereich scheinen aber wegen der weitgehenden Verschlossenheit diplomatischer Dienste gegenüber Außenstehenden klare Grenzen gesetzt zu sein (Wilke 1980, S. 8-9). Die vorliegende Untersuchung ist ein Schritt, um den individuellen Karriereerfolg dieser oft als abgehoben wahrgenommenen Entscheidungselite zu deuten und dabei den Stellenwert einzelner theoretischer Ansätze zu evaluieren. Wir beziehen uns dabei auf vier Kohorten von Diplomaten im höheren Auswärtigen Dienst (AD).

Die Erklärungen dafür, warum Diplomaten gegen Ende ihrer Laufbahn gehaltsmäßig auf unterschiedlichen Niveaus stehen, entnehmen wir dem Sozialisationsansatz, der im Konstruktivismus verwendet wird und der aus der Sozialpsychologie stammt, sowie dem Rational-Choice-Ansatz. Wir leiten insgesamt sechs Hypothesen aus diesen zumindest teilweise widerstreitenden Theorien $a b$ und überprüfen diese anhand von Daten aus einer

1 Normalerweise ist die Bezeichnung „Diplomat" den Beamten des Auswärtigen Diensts im Ausland vorbehalten. Zur Vereinfachung wird dieser Begriff hier auch für jene im Inland verwendet. 
schriftlichen Befragung von vier diplomatischen „Crews“ ${ }^{\text {“2 }}$ (Eintrittsjahrgänge 19701973) mithilfe einer ordinalen logistischen Regression.

Die Ergebnisse der statistischen Analyse wie auch von qualitativen Leitfadengesprächen deuten darauf hin, dass der Berufserfolg im höheren $\mathrm{AD}$ hauptsächlich auf den Mechanismus der strategischen Nutzenkalkulation zurückführen ist. Es sind eher Netzwerkarbeit und Konformismus als Sozialisationsvariablen (familiärer Hintergrund, politische Gesinnung), die darüber entscheiden, mit welcher Besoldungsstufe ein Diplomat aus dem Dienst ausscheidet. Die Zusammensetzung der untersuchten Diplomatenlehrgänge deutet jedoch darauf hin, dass Gruppen- oder Sozialisationsmerkmale vorwiegend zur Erklärung der Selbstrekrutierung bzw. der Rekrutierung im Vorfeld der beruflichen Karriere beitragen.

\section{Theorie}

\subsection{Rational Choice versus Sozialisation}

Welche Faktoren verhelfen Diplomaten zum Karriereerfolg? Die theoretische Grundlage zur Erörterung dieser Frage bilden zwei sozialwissenschaftliche Theorien: der Sozialisationsansatz, der aus der Psychologie und Soziologie kommt, sowie der Rational-ChoiceAnsatz. Nach einer kurzen Darstellung der Hauptmerkmale dieser Ansätze soll auf das Problem eingegangen werden, ob und wie sich diese Erklärungsmodelle voneinander abgrenzen lassen.

Sozialisation ist seit Jahrzehnten ein zentrales Konzept der Entwicklungspsychologie und der Politischen Soziologie; in den vergangenen Jahren ist es auch innerhalb des politikwissenschaftlichen Konstruktivismus zu einer zentralen Analysekategorie geworden. Sozialisation bezeichnet allgemein einen Vorgang, bei dem sich Akteure durch soziale Interaktion bestimmte Normen, Verhaltens- und Denkweisen aneignen (vgl. Checkel 2005). Natürlich sozialisieren sich Individuen ihr Leben lang. Wichtig für diesen graduellen Prozess ist das Zusammenspiel zwischen dem Individuum mit seinen körperlichen und psychischen Grundmerkmalen und seiner physikalischen und sozialen Umwelt (Hurrelmann 2006, S. 7).

Sozialisationsprozesse werden durch Sozialisationsinstanzen gesteuert, also bestimmte Personen, Gruppen, Organisationen, Staaten etc., die je nach Lebensphase mit unterschiedlicher Intensität auf das Individuum einwirken (Hurrelmann 2006, S. 32-33). In der Literatur wird oftmals zwischen verschiedenen Sozialisationsmechanismen unterschieden. Dabei geht es um die kausalen Mechanismen, die auf der Individualebene zur Annahme von bestimmten Normen, Regeln und Verhaltensweisen im Zusammenhang mit einer Sozialisationsinstanz führen (Johnston 2001, S. 496-499; Zürn u. Checkel 2005, S. 1049).

Für das Verständnis der Sozialisationshypothesen, die wir entwickeln, ist die grundlegende Annahme dieses Ansatzes wichtig, derzufolge die Sozialisation einen Mikropro-

2 Die Diplomatenlehrgänge tragen seit der Weimarer Republik inoffiziell die Bezeichnung "Crew". 
zess darstellt, der unabhängig von materiellen Anreizen abläuft. Deshalb stellt sich die Frage, wie das entwicklungspsychologische Grundkonzept zur Erklärung von Karriereerfolg beitragen kann. Im Zusammenhang mit beruflichen Karrieren lässt sich das Sozialisationsergebnis vor allem auf jene Werte, Normen und Verhaltensstandards reduzieren, die notwendig sind, um eine berufliche Rolle zu erfüllen. Sozialisation bestimmt, wie gut sich das Individuum in dieser Rolle zurechtfindet und bis zu welchem Grad es das im Beruf erwünschte Verhalten erlernt und aufweist (Van Maanen u. Schein 1979, S. 3-7; Chow 2002, S. 720).

Die Theorie der rationalen Entscheidung, in den deutschsprachigen Sozialwissenschaften auch als Rational-Choice-Theorie bekannt, rückt im Gegensatz zu Sozialisationsansätzen die materiellen Anreize in den Vordergrund, an denen sich individuelle Entscheidungsträger und somit auch Diplomaten orientieren. Die Fokussierung auf solche extrinsischen Handlungsmotivationen ist innerhalb des Rational-Choice-Ansatzes nicht unumstritten. Viele Autoren gehen etwa davon aus, dass sowohl materielle als auch nicht-materielle Anreize wie Normen, Werte oder Gefühle Teil der individuellen Nutzenfunktion sind (Chong 2000; Elster 1989; Kato 1996; Boudon 1998). Dies entspricht dem „thin rationalism", der die Frage nach den Komponenten der Nutzenfunktion offen lässt (Ferejohn 1991, S. 282; Yee 1997). Da diese Version der Nutzenfunktion zu Tautologien führen kann und sich nicht eindeutig von dem Sozialisationskonzept abgrenzen lässt, soll hier von einem ,thick rationalism" ausgegangen werden (Hooghe 2001, S. 20). Es wird unterstellt, dass primär materielle Anreize und Sanktionen individuelle Präferenzen und Verhaltensweisen steuern.

Lange wurden der Sozialisations- und der Rational-Choice-Ansatz als konkurrierend und unvereinbar angesehen. Seit einigen Jahren geht die Tendenz jedoch dahin, die Trennlinien zwischen den zwei Ansätzen verschwimmen zu lassen und die beiden theoretischen Perspektiven als komplementär zu betrachten (Aspinwall u. Schneider 2000; Chong 2000; Hooghe 2001, 2005; Fearon u. Wendt 2002; Jupille et al. 2003; Johnston 2005; Zürn u. Checkel 2005).

Die Umwelt, in der Individuen agieren, ist sowohl sozial als auch materiell beschaffen (Jupille et al. 2003, S. 14). Auf der einen Seite eignet sich das Individuum durch Sozialisationsprozesse und durch die Interaktion mit den jeweiligen Sozialisationsinstanzen über die Zeit hinweg bestimmte Prädispositionen an. Diese gelten als rigide und stabil und spiegeln sich in Charakterzügen und Fähigkeiten, der Ideologie, der Parteipräferenz etc. wider (Sears u. Funk 1991; Chong 2000). Auf der anderen Seite bieten sich dem Individuum im Laufe seines Lebens materielle Anreize, die mittels strategischer Kalkulation zur Veränderung von Einstellungen und Verhaltensweisen führen können.

Man kann also nicht von einer Entweder-oder-Logik ausgehen, sondern davon, dass individuelles Handeln und Präferenzen meist einer Kombination der beiden Mechanismen entspringen. Dies schließt nicht aus, dass einer der Ansätze unter bestimmten Umständen eine höhere Erklärungskraft besitzt. Es hängt von den jeweiligen Rahmenbedingungen ab, ob Sozialisationsmechanismen oder strategische Kalküle überwiegen. Hooghe (2001, S. 214; 2005, S. 870-871) stellt in ihren Studien zu Beamten der Europäischen Kommission beispielsweise fest, dass strategische Erwägungen dann überwiegen, wenn die individuelle Karriere auf dem Spiel steht, also dann, wenn die Individuen einen direkten Zusammenhang zwischen ihren Präferenzen und ihrer Karriere erkennen. 
Dieser Aufsatz stellt keinen allgemeinen Test der Sozialisationstheorie gegen deren rationalistische „Konkurrentin“ dar. Da beide Theorien bereits in vielen empirischen Arbeiten ihre Erklärungskraft bewiesen haben, würde ein solcher Test keine fruchtbaren Ergebnisse erbringen. Vielmehr wollen wir prüfen, welche spezifischen Faktoren den Karriereerfolg von Diplomaten bestimmen. Um diese Determinanten dennoch zu verorten, entwickeln wir zwei unterschiedliche, wenn auch nicht völlig disjunkte Erklärungsmodelle.

\subsection{Hypothesen}

Es wurden bereits einige Untersuchungen zu den Karrierefaktoren im deutschen öfentlichen Dienst durchgeführt (Luhmann u. Mayntz 1973; Steinkemper 1974; Pippke 1975; Dreher 1996). Allerdings ist die empirische Forschung in diesem Bereich veraltet und basiert lediglich auf bivariaten Tests. Um mögliche Erfolgsfaktoren zu identifizieren, wurden im Vorfeld dieser Untersuchung explorative Leitfadeninterviews mit sieben Angehörigen des höheren AD durchgefuihrt. Dabei haben sich sechs Forschungshypothesen herauskristallisiert, die den konkurrierenden theoretischen Erklärungsmodellen zugeordnet werden. Die ersten zwei lassen sich dem Sozialisationsmodell und die letzten drei dem Rational-Choice-Modell zuordnen. Hypothese 3 lässt keine klare Zuordnung zu, weshalb diese als „Hybrid-Hypothese" bezeichnet wird.

Amtsbonus, Parteimitgliedschaft und Juristenmonopol: Söhne und Töchter aus Diplomatenfamilien (,Amtskinder's) verfügen über besondere Sozialisationserfahrungen. Sie lernen bereits in ihrer Kindheit den Umgang mit neuen Kulturen, Sprachen und wechselnden Umgebungen. Ferner kommen sie früh mit politischen Themen in Berührung und eignen sich einen speziellen sozialen Habitus an. Nach Schubert (2006, S. 43) fallen darunter "Charakterzüge und Eigenschaften, die in der Persönlichkeit verankert sind" und die auf der Verinnerlichung im Verlauf der primären Sozialisation basieren. Hinzu kommt, dass Amtskinder eher als andere wissen, welche Verhaltensweisen der Karriere im AD zuträglich sind und welche nicht. Es wird angenommen, dass Amtskindern die Übernahme der beruflichen Rolle dadurch leichter fällt als ihren Kollegen im AD. Außerdem ist zu vermuten, dass sie Zugang zu laufbahnfördernden Netzwerken innerhalb des $\mathrm{AD}$ haben. Diplomatenkinder verfügen also durch ihre Sozialisation im Rahmen von Diplomatenfamilien über einen Informations- und Erfahrungsvorsprung und eventuell über ein karriereförderliches Netzwerk.

\section{HI: Amtskinder haben höhere Erfolgschancen im Auswärtigen Dienst.}

Verschiedene Studien belegen die steigende Parteipolitisierung der deutschen Verwaltung im Laufe der letzten 40 Jahre (Steinkemper 1974; Derlien 1988, 2003; Pippke 1975; Mayntz u. Derlien 1989; Schwanke u. Ebinger 2006). ${ }^{3}$ Obwohl sich der AD als überparteilich darstellt, gibt es Beispiele dafür, dass in die Personalpolitik gelegentlich parteipolitische Überlegungen einfließen. So wurden im Jahr 1999 auf Antrag der Regierungsfraktionen SPD/Die Grünen trotz allgemeiner Stellenstreichungen vier höherran-

3 In dieser Untersuchung liegt der Schwerpunkt auf der formalen Politisierung, insbesondere auf der Parteipolitisierung (Rouban 2003, S. 313-317). Die Parteipolitisierung bezieht sich auf Anstellung, Beforderung oder Abberufung von Beamten aufgrund ihrer Parteimitgliedschaft. 
gige Planstellen im höheren $\mathrm{AD}$ eingerichtet, die durch politische Quereinsteiger besetzt werden sollten (Biehler 2000, S. 2400-2402; Weigert 2004, S. 163-167). Gleichermaßen hat das Kanzleramt 2007 sein Veto eingelegt, als der Botschafterposten in Warschau mit einem SPD-nahen Diplomaten besetzt werden sollte (Beste 2007).

Die externe Einflussnahme von Parteigremien auf die Personalpolitik ist wahrscheinlich damit zu begründen, dass die außenpolitische Spitze, also der Bundeskanzler und Außenminister, sich mit Diplomaten umgibt, von denen sie die größtmögliche politische Loyalität erwarten kann. Wenn andere ausschlaggebende Entscheidungsmerkmale fehlen, stellt die Mitgliedschaft in der gleichen Partei ein einschlägiges Kriterium dar.

Ob sich eine Parteimitgliedschaft als karriereförderlich erweist oder nicht, hängt dementsprechend von der jeweiligen politischen Konstellation ab. Während des Untersuchungszeitraums (1970-2008) stellte die SPD bzw. die CDU den Bundeskanzler, das Amt des Außenministers teilten sich FDP, die Grünen und die SPD. Daher wird hier die Mitgliedschaft in einer dieser vier Parteien als karriereförderlich betrachtet. Dies sowie die Tatsache, dass es im AD bestimmte parteinahe Gruppierungen (Freundeskreise etc.) mit Netzwerkcharakter gibt, führen zu folgender Hypothese:

H2: Die Mitgliedschaft in einer der Regierungsparteien erhöht die Erfolgschancen im Auswärtigen Dienst.

Hiermit ist noch nichts dazu gesagt, welchem theoretischen Lager Hypothese 2 zuzuschlagen ist. Allgemein wird die Parteizugehörigkeit eher als Ergebnis von Sozialisationsprozessen angesehen (Steinkemper 1974; Markus 1979; Sears u. Funk 1991, S. 74; Hooghe 2001). Sie spiegelt demnach ein grundlegendes Einstellungsmuster wider, welches sich bereits seit der frühen Kindheit entwickelt und in den Erwachsenenjahren zeitlich relativ stabil ist. Dennoch könnte man vermuten, dass Opportunisten im AD einer Partei beitreten oder ihr Parteibuch wechseln, um ihre Karrierechancen zu erhöhen. Bisherige Studien haben dies aufgrund der hohen Durchschnittsdauer der Parteimitgliedschaften verneint (Mayntz u. Derlien 1989, S. 392; Derlien 1994, S. 270; 2003, S. 410). Auch hier wird davon ausgegangen, dass die Parteimitgliedschaft im $\mathrm{AD}$ ein zeitlich relativ stabiles Merkmal ist, welches nur in Ausnahmefällen von Opportunismus zeugt. Hypothese 2 wird somit dem Sozialisationsmodell zugeordnet.

Der höhere $\mathrm{AD}$ steht für Hochschulabsolventen aller Fachrichtungen offen. Dennoch zeichnet er sich, so wie die deutsche Verwaltung allgemein, durch ein langjähriges „Juristenmonopol“ aus. In den Diplomatenlehrgängen von 1950 bis 1973 belief sich der Juristenanteil beispielsweise durchschnittlich auf $68 \%$, wobei kein rückläufiger Trend festzustellen war (Curtius 1974). Es stellt sich jedoch die Frage, ob dieses quantitative Juristenmonopol auch qualitativer Natur ist. Sind Juristen nur zahlenmäßig überrepräsentiert, oder machen sie auch eher Karriere?

Auf diese Interviewfrage antworteten Diplomaten, dass der Arbeits- und Analysestil sowie das Berichtsverfahren im höheren $\mathrm{AD}$ nach wie vor durch einen juristischen Stil geprägt seien. Juristen wurde daher ein gewisser Startvorteil gegenüber den Absolventen anderer Studienfächer zugesprochen, die entsprechende Kenntnisse und Arbeitsweisen erst erlernen müssen. Pippke (1975, S. 137) spricht in diesem Zusammenhang von einer „Prägung der Arbeitsfelder durch ihre generationenlange Besetzung mit Juristen, die zu einer zunehmenden Justitialisierung der Verwaltung und einer Hypertrophie des lega- 
listischen Denkens in ihr geführt haben" ${ }^{4}{ }^{4}$ Ein weiterer und abschließender Vorteil der Juristen entspringt dem „Kooptationscharakter von Beförderungen“, also der Tendenz von Entscheidungsträgern, eher jene Individuen zu befördern, die ihnen im Hinblick auf bestimmte Merkmale (in diesem Fall das Jurastudium) ähnlich sind (Pippke 1975, S. 137).

H3: Juristen haben höhere Erfolgschancen im Auswärtigen Dienst.

Im Fall des Jurastudiums stellt sich die Zuordnung zu einem der theoretischen Ansätze als besonders schwierig dar. Man könnte annehmen, dass ein Jurastudium gewählt wird, weil man von vornherein plant, in den Staatsdienst zu gehen, und weiß, dass diese Studienrichtung die besten Karrierechancen verspricht (vgl. Steinkemper 1974, S. 19). Dies würde bedeuten, dass das Individuum bereits vor Studienbeginn die Vor- und Nachteile eines Jurastudiums für die spätere Karriere abwăgt und daraufhin seine Entscheidung trifft. Allerdings kann nicht davon ausgegangen werden, dass die nötigen Informationen für eine solche Kosten-Nutzen-Kalkulation im Hinblick auf ein bestimmtes Berufsziel (klare payoffs eines Jurastudiums) schon so früh vorliegen (Apel 1993, S. 130). Es kann beispielsweise genauso gut sein, dass das Individuum aus einer Juristenfamilie stammt und aufgrund der Wertebildung in diesem familiärem Umfeld diese Fachrichtung einschlägt. Ein solches Hineinwachsen in die Rechtswissenschaften und ihr Weltbild wäre eher dem Sozialisationsansatz zuzuordnen.

Bisherige Untersuchungen zu Determinanten der Studienfachwahl haben in dieser Hinsicht keine eindeutigen Ergebnisse geliefert. Die extrinsische Motivation hat sich ebenso wie die intrinsische Motivation, der familiäre Hintergrund, das Geschlecht und die schulische Leistung als erklärende Variable bewährt (Apel 1993; Heublein u. Sommer 2002). Da für die Untersuchungsgesamtheit keine Daten zu den Gründen der Studienfachwahl vorliegen, kann man Hypothese 3 nicht eindeutig dem Sozialisationsansatz oder dem Rational-Choice-Ansatz zuordnen. Aus diesem Grund wird sie als Hybrid-Hypothese bezeichnet und auch im Rahmen der statistischen Analyse als solche behandelt.

Machtnähe und Konformismus: Üblicherweise verbringen Diplomaten zwei Drittel ihrer Dienstzeit im Ausland und ein Drittel in der Zentrale, also im Auswärtigen Amt (AA) (Brandt u. Buck 2003, S. 254). Auf die Frage, wo man Karriere macht - in der Zentrale oder im Ausland -, antworteten alle Interviewpartner spontan, dass die Karriere im Inland gemacht werde. Der wichtigste Grund hierfür scheint die Nähe zum Leitungsbereich und zu den politischen Entscheidungsträgern und die damit einhergehende „Sichtbarkeit" zu sein. In der Zentrale werden besonders hohe Ansprüche an Leistungsbereitschaft und -fähigkeit gestellt. ${ }^{5}$ Fleiß und fachliche Leistung können dort erheblich besser beurteilt werden als in einer weit entfernten Auslandsvertretung.

4 Tatsächlich verfügt der AD über eine Vielzahl von Abteilungen mit juristischem Schwerpunkt (Abteilung 5 im Inland oder Rechts- und Konsularabteilungen in den Auslandvertretungen). Für einige Aufgaben wie etwa notarielle Tätigkeiten benötigt man ebenfalls richterliche Befugnisse.

5 In den Interviews war die Rede von bis zu 70 Wochenstunden und gegebenenfalls auch Verfügbarkeit an den Wochenenden. 
Außerdem haben die Beamten nur in der Zentrale die Möglichkeit, politische Entscheidungen mitzugestalten und zu formulieren. Dadurch haben sie häufig einen entscheidenden Informationsvorsprung. In einigen Interviews wurde vom Karrierefaktor „Glück“ bzw. von günstigen, zufälligen Konstellationen gesprochen, die zum Karriereerfolg verhelfen. ${ }^{6}$ Solche Umstände, die das Knüpfen informeller Kontakte („,Netzwerkarbeit") umfassen können, ergeben sich auch eher im Inland. Im Zweifelsfall läuft man dem Staatssekretär oder dem Chef der Personalabteilung in der Zentrale und nicht in der Auslandsvertretung über den Weg (vgl. dazu Dreher 1996, S. 448).

Die Arbeit in der Zentrale führt also zu einer höheren Sichtbarkeit persönlicher Leistungen, einer besseren Einbindung in den Informationsfluss sowie einer stärkeren personellen Vernetzung mit der Leitungsebene.

H4a: Diplomaten, die überdurchschnittlich viel Zeit auf Dienstposten in der Zentrale verbringen, haben höhere Erfolgschancen im Auswärtigen Dienst.

In einem zweiten Schritt stellt sich die Frage, ob diese Hypothese für alle Dienstposten in der Zentrale gleichermaßen zutrifft. Während der Interviews wurden folgende Arbeitsbereiche oder Funktionen als besonders karriereförderlich bezeichnet: Ministerbüro (AA), Leitungsstab, Planungsstab, Persönlicher Referent der Staatssekretäre oder Staatsminister, Bundeskanzleramt und Bundespräsidialamt. Diese machtnahen Arbeitsbereiche zeichnen sich klar durch ihre Leitungsnähe sowie ihren inhaltlichen Schwerpunkt auf der konzeptionellen Ebene der Außenpolitik aus. Es wird vermutet, dass die oben genannte Sichtbarkeit, die personelle Verknüpfung und die Einbindung in den Informationsfluss in diesen machtnahen Arbeitsbereichen eher zum Tragen kommen als in anderen Arbeitsbereichen des AA. Daraus ergibt sich folgende Hypothese:

$H 4 b$ : Dienstliche Verwendungen in machtnahen Arbeitsbereichen erhöhen die Erfolgschancen im Auswärtigen Dienst.

In den Interviews fiel auf, dass Diplomaten sich der Karrierewirksamkeit der Arbeit in der Zentrale durchaus bewusst sind. Weiter wurde deutlich, dass sie sich aktiv für eine längere Verweildauer in der Zentrale einsetzen können und dass sie auch - bei entsprechender Eignung - einen gewissen Einfluss auf die Wahl des Arbeitsbereichs in der Zentrale haben. Die Bedingungen für eine Kosten-Nutzenkalkulation sind in diesem Fall also gegeben. Es wird angenommen, dass karrierebewusste Diplomaten eine entsprechend strategische Postenwahl betreiben und mehr als das übliche Drittel ihrer Berufsarbeitszeit in der Zentrale verbringen. Die Hypothesen $4 \mathrm{a}$ und $4 \mathrm{~b}$ werden dem Rational-ChoiceModell zugeordnet.

Beamte unterliegen einer gesetzlichen Neutralitäts- und Mäßigungspflicht. Sie sind „,ihrem Arbeitgeber gegenüber zur Treue verpflichtet und haben sich nach besten Kräften für dessen Interessen einzusetzen" (BMI 1984, S. 147). Diese Regelungen zielen vor allem darauf ab, die Kontinuität der Verwaltung bei wechselnden politischen Prinzipalen zu garantieren (Steinkemper 1974, S. 46). Die Funktionsfähigkeit und Stabilität des AD hängen aufgrund der „Verstreutheit der Organisation“ (End 1969, S. 55) besonders

6 In Pippkes Untersuchung (1975, S. 182) haben 29,1\% der Spitzenpositionsinhaber im öffentlichen Dienst „Begünstigung durch Zufall" als wichtigen Erfolgsfaktor angegeben. 
von der Loyalität seiner Angehörigen ab. Diplomaten vertreten die Interessen Deutschlands im Ausland, und ihre Entscheidungen und Tätigkeiten können durch die Zentrale höchstens im Nachhinein und unter Prestigeverlust rückgängig gemacht werden (Wilke 1980, S. 106). Umso wichtiger ist es auch, dass eine klare Trennung von ,privater" und „offizieller" Meinung stattfindet.

In der Untersuchung von Luhmann u. Mayntz (1973, S. 244-245) wurde Loyalität neben der allgemeinen Leistungsorientierung von den befragten Beamten als wichtigstes beförderungsrelevantes Merkmal im öffentlichen Dienst angesehen. Bei einer Befragung deutscher und britischer Beamten zu Erfolgskriterien im öffentlichen Dienst (Nolterieke 1981, S. 151-169) hingegen haben deutsche Beamte in erster Linie die Eigenschaften „Opportunismus und Anpassungsfähigkeit“ angegeben.

Doch wo ist die Grenze zwischen Loyalität und Opportunismus? „Loyalität heißt, die eigene Meinung zugunsten der Regierungsmeinung bei der Arbeit hintan zu stellen“ (Interview). Bei der Loyalität geht es also eher um die offizielle Meinung der Diplomaten. Opportunismus hingegen beschreibt die Änderung bzw. Anpassung von privaten Überzeugungen und Verhaltensweisen aufgrund von Nützlichkeitserwägungen (Wenninger 2001, S. 187). Dies legt für die Welt der Diplomatie die Annahme nahe, dass opportunistische Repräsentanten des AD ihre politischen Präferenzen nur so lange beibehalten, wie diese ihrer Karriere zugutekommen (vgl. Hooghe 2001, S. 14). Bei einem Regierungswechsel könnte dementsprechend eine Anpassung der Einstellungen stattfinden.

Opportunismus ist ein negativ besetzter Begriff, der oft mit Charakterlosigkeit und Persönlichkeitsschwäche assoziiert wird (Wenninger 2001, S. 187). Aus Respekt vor den Untersuchungspersonen soll fortan die Bezeichnung Konformismus ${ }^{7}$ verwendet werden. In dieser Untersuchung werden Diplomaten dementsprechend in zwei Gruppen eingeteilt: Konformisten, die ihre Einstellung an die jeweilige Regierung anpassen, und Prinzipientreue, die weitgehend an ihren persönlichen Einstellungen festhalten.

Zusammenfassend lässt sich sagen, dass die Loyalität zum Dienstherrn eine wichtige Voraussetzung für die Funktionsfähigkeit des $\mathrm{AD}$ und somit auch grundlegend für die Diplomatenkarriere ist. Kalkulierende Diplomaten sind sich dessen bewusst und orientieren sich an der außenpolitischen Linie des Dienstherrn:

H5: Konformisten, die ihre Einstellung nach der jeweiligen Regierung richten, haben höhere Erfolgschancen im Auswärtigen Dienst.

\section{Forschungsdesign und Methode}

Den Autoren sind zum Personal im AD keine öffentlich zugänglichen Individualdaten bekannt, die sich für einen systematischen Hypothesentest eignen würden. Aus diesem Grund haben wir einen schriftlichen Fragebogen entworfen, der an vier ausgewählte

7 Wenninger (2001, S. 374) definiert Konformismus als „Haltung, die stets um Anpassung der persönlichen Einstellungen an die bestehenden Verhältnisse bemüht ist." 
Diplomatenlehrgänge versandt wurde. ${ }^{8}$ Dabei handelt es sich um jene Berufseinstiegskohorten, die zwischen 1970 und 1973 mit dem Vorbereitungsdienst für den höheren AD begonnen haben. ${ }^{9}$ Da die abhängige Variable Karriereerfolg ist, wurden die Crews so gewählt, dass ihre Mitglieder entweder kürzlich in den Ruhestand traten oder sich in ihrer letzten dienstlichen Verwendung befanden. Der Vorteil dieser Fallauswahl ist, dass ähnliche organisatorisch bedingte Karrieremöglichkeiten (z. B. Stellenkegel) vorausgesetzt werden konnten und vollständige berufliche Laufbahnen vorliegen. Hinzu kommt, dass die Untersuchungsobjekte im Bezug auf die Merkmale Alter, Dienstalter und Eintrittsjahr keine große Varianz aufweisen, sodass man Generationeneffekte (Mannheim 1928/29) ausschließen kann.

Die vier untersuchten Kohorten umfassten ursprünglich 133 Personen, wovon 16 zum Untersuchungszeitpunkt bereits verstorben waren und sechs frühzeitig aus dem $\mathrm{AD}$ ausgetreten sind. Während drei der sechs Ausgeschiedenen entweder als Attaché oder direkt nach ihrer Attachézeit ausgetreten sind, haben die anderen drei zu verschiedenen Zeitpunkten ihrer diplomatischen Laufbahn den Beruf gewechselt, um ihre Karriere anderswo weiterzuführen. Erstere werden in dieser Untersuchung nicht betrachtet, da bei ihnen keine Informationen zur diplomatischen Laufbahn vorliegen. An diejenigen, die in der Mitte ihrer Laufbahn ausgeschieden sind, wurde eine abgeänderte Version des Fragebogens versandt. ${ }^{10}$

Im Falle derer, die während des Erhebungszeitraums noch im Dienst waren, ließen sich die Kontaktdaten über offizielle Quellen im Internet erschließen. Die Kontaktdaten der sich im Ruhestand befindenden Diplomaten wurden zum einen über Internetrecherchen und Telefonbücher, zum anderen über persönliche Kontakte anhand des Schneeballverfahrens (vgl. Schnell et al. 1999, S. 280) ermittelt.

Aus den verschiedenen Quellen ergaben sich 88 Post- und 82 Emailadressen. Für 13 pensionierte Diplomaten lagen keinerlei Kontaktdaten vor. Letztendlich konnte der Fragebogen an 102 Diplomaten geschickt werden. Davon haben sich insgesamt 51 an der Befragung beteiligt.

\subsection{Operationalisierung}

Die meisten Variablen dieser Untersuchung ergeben sich direkt aus den Items des Fragebogens (s. Online-Anhang). Für einige mussten jedoch spezielle Korrespondenzregeln

8 Um die Güte des Erhebungsinstruments zu testen und seine Validität zu erhöhen, wurden zwei Pretests durchgeführt.

9 Bei der Fallauswahl wurden weder Seiteneinsteiger noch Laufbahnaufsteiger berücksichtigt. Da die Anzahl der Seiteneinsteiger in der Verwaltungselite jedoch relativ niedrig ist (Derlien u. Pippig 1990, S. 105) und davon ausgegangen werden kann, dass Laufbahnaufsteiger keine wirkliche Konkurrenz für die Untersuchungspersonen darstellen, werden keine Verzerrungen aufgrund der genannten Einschränkungen erwartet. Dennoch soll an dieser Stelle betont werden, dass sich die Aussagen dieses Artikels lediglich auf reine Laufbahnbeamte des höheren AD beziehen.

10 Zwei der angeschriebenen Personen sind vorzeitig aus dem AD ausgeschieden und tauchen so nicht in der statistischen Analyse auf, die wir hier präsentieren. 
(Schnell et al. 1999, S. 125) aufgestellt werden. Auf Letztere soll im Anschluss vertieft eingegangen werden.

Abhängige Variable: Um zu erklären, warum einige Diplomaten erfolgreicher sind als andere, benötigt man ein valides Maß für Berufserfolg. In der Organisationsliteratur wird oftmals zwischen dem subjektiven und dem objektiven Berufserfolg unterschieden (Hall 1987; Judge et al. 1995; Seibert et al. 2001). Der subjektive Berufserfolg wird anhand intrinsischer Karrieremaße, also der Zufriedenheit und Erfüllung des Individuums hinsichtlich seiner beruflichen Laufbahn, gemessen. Im Falle des AD kann dies beispielsweise bedeuten, in einem bevorzugten Themenbereich (z.B. Kulturpolitik) zu arbeiten oder bestimmte, persönlich als interessant empfundene Posten zu besetzen. Der objektive Karriereerfolg hingegen wird anhand von beobachtbaren Indikatoren (extrinsische Karrieremaße) wie Gehalt, Anzahl der Beförderungen, Rangstufe etc. gemessen (London u. Stumpf 1982).

Da sich die Bestimmungsfaktoren für den subjektiven und für den objektiven Karriereerfolg wesentlich voneinander unterscheiden (Judge et al. 1995), aber auch, um eine bessere Vergleichbarkeit herzustellen, wird der Berufserfolg hier ausschließlich durch ein extrinsisches Karrieremaß - die Besoldungsgruppe - operationalisiert. Im höheren AD gibt es acht verschiedene Besoldungsgruppen. Diplomaten beginnen ihre Karriere mit A13 und dem Titel „Legationssekretär" bzw. „Legationsrat". Steigt der Beamte bis zur Spitze der Hierarchie, also der Position des Staatssekretärs auf, so durchläuft er typischerweise die folgenden Besoldungsgruppen: A14 (Legationsrat I. Klasse), A15 (Vortragender Legationsrat), A16/B3 (Vortragender Legationsrat I. Klasse), B6 (Ministerialdirigent), B9 (Ministerialdirektor) und schließlich B11 (Staatssekretär) (vgl. BBesO A/B, S. 1975). Diese Dienstgrade oder Besoldungsgruppen geben entsprechend der jeweiligen Besoldungsordnung die Grundgehälter an. ${ }^{11}$

Die Besoldungsgruppe und der hierarchische Rang hängen im $\mathrm{AD}$ - wie in anderen Bundesministerien - strikt zusammen. So ist der Vortragende Legationsrat I. Klasse (A16 oder B3) in der Regel dazu befugt, Referatsleiter in der Zentrale zu werden; der Ministerialdirigent Unterabteilungsleiter und der Ministerialdirektor Abteilungsleiter (Schütz 1965). Auch die Besetzung von Leiterposten im Ausland ist eng mit der Besoldungsgruppe verknüpft. Ein Diplomat kann bereits als Vortragender Legationsrat Leiter einer kleineren Botschaft oder eines Generalkonsulats werden. Generell gilt aber: je größer und wichtiger die Auslandsvertretung, desto höher die Besoldungsgruppe.

In der Literatur zu Karrieren im öffentlichen Dienst wurde oftmals zwischen der Anzahl der Beförderungen (oder dem Erreichen von Spitzenpositionen) und der Beförderungsgeschwindigkeit unterschieden (Luhmann u. Mayntz 1973; Pippke 1975; Weigert 2004). Sowohl Luhmann u. Mayntz (1973, S. 173) als auch Pippke (1975, S. 174) stellen jedoch fest, dass die Beförderungsgeschwindigkeit in erster Linie von strukturellen Merkmalen des Beförderungssystems abhängt. Abgesehen davon, scheinen die Bestimmungsfakto-

11 Während der Dienstzeit kommen für die Beamten im Ausland noch die jeweiligen Zulagen (Zonenstufe, Aufwandsentschädigung, Kaufkraftausgleich etc.) dazu. Aus diesem Grund ist das Gehalt eines Beamten im Ausland meist wesentlich höher als das eines Beamten im Inland mit gleicher Besoldungsgruppe. Da diese Zulagen je nach Posten variieren, scheint das Grundgehalt jedoch das am besten vergleichbare Maß für den Berufserfolg zu sein. 
ren für die Anzahl der Beförderungen und die Beförderungsgeschwindigkeit wesentlich dieselben zu sein. Diejenigen, die am erfolgreichsten sind, weisen gleichzeitig auch die höchste Beförderungsgeschwindigkeit auf (Dreher 1996, S. 640). Karriereerfolg wird in dieser Untersuchung durch die Summe der Beförderungen innerhalb der gesamten Laufbahn operationalisiert. Dementsprechend ist der Indikator für Berufserfolg die Besoldungsgruppe, mit der die Diplomaten aus dem $\mathrm{AD}$ ausscheiden (fortan: pensionsrelevante Besoldungsgruppe). Zur Bestimmung der pensionsrelevanten Besoldungsgruppe wurde nach einer Gesamtauflistung der Dienstposten gefragt. Die dazugehörigen Besoldungsgruppen finden sich im Einzelplan 05 des Bundeshaushaltsplans.

Die abhängige Variable Berufserfolg wird also anhand des extrinsischen Erfolgsmaßes, der pensionsrelevanten Besoldungsgruppe, gemessen. Diese gibt das Endgrundgehalt der Beamten an und ist gleichzeitig ein Indikator für die Anzahl der Beförderungen. Die abhängige Variable ist ordinalskaliert, da die Abstände zwischen den Besoldungsgruppen weder hinsichtlich der Anzahl der Planstellen ${ }^{12}$ noch hinsichtlich der jeweiligen Gehälter als gleich betrachtet werden können. Die abhängige Variable umfasst die Besoldungsgruppen A15 bis B9. ${ }^{13}$ Aufgrund der niedrigen Fallzahl wurden die Besoldungsgruppen A15 bis B3 zusammengelegt; somit hat die abhängige Variable drei Ausprägungen.

Unabhängige Variablen: Zur Messung des Konzepts Amtskinder wurde im Fragebogen danach gefragt, ob Mutter oder Vater des/r Befragten im AD tätig war. Diplomaten, die in einer Diplomatenfamilie sozialisiert wurden, erhalten den Wert $1 .{ }^{14}$ Allen anderen wird der Wert 0 zugeordnet. Die Variable Parteimitgliedschaft wurde über zwei Fragen erhoben. Zunächst erfragten wir, ob die Untersuchungsperson Mitglied einer politischen Partei ist oder war. Ferner erhoben wir die Parteineigung. Für Parteimitglieder gingen wir dabei davon aus, dass sie auch die entsprechende Partei in Frage 24 ankreuzen. Nun besteht die Möglichkeit, dass eine Untersuchungsperson die Frage nach Parteimitgliedschaft zwar positiv beantwortet, aber keine Angabe zur Parteineigung macht. In diesem Fall kann davon ausgegangen werden, dass sie Mitglied einer der Regierungsparteien im Untersuchungszeitraum ist bzw. war. ${ }^{15}$ Die dichotome Variable Parteimitgliedschaft gibt demnach an, ob eine Untersuchungsperson zu irgendeinem Zeitpunkt während ihrer Laufbahn einer der Regierungsparteien angehört hat (1) oder nicht (0). Die Dummy-Vari-

12 Der Stellenkegel des AD ist pyramidenförmig. Die Anzahl an Planstellen nimmt mit höheren Besoldungsgruppen $\mathrm{ab}$.

13 A15 ist die niedrigste erreichte Besoldungsgruppe in der Stichprobe. Die Person, die die Besoldungsgruppe $\mathrm{B} 11$ erreicht hat, hat sich nicht an der Befragung beteiligt.

14 Die Befragten sollten den Gesamtzeitraum der Tätigkeit ihres Elternteils im AD angeben. Wenn der Elternteil nur wenige Jahre Angehöriger des $\mathrm{AD}$ war, oder zu einem Zeitpunkt, an dem die Person bereits das Elternhaus verlassen hat, wurde diese nicht als Amtskind kodiert.

15 Die Regierungs- bzw. Koalitionsparteien im Untersuchungszeitraum sind: CDU/CSU, SPD, FDP und Bündnis 90/Die Grünen. Es ist anzunehmen, dass die Parteimitglieder in der Untersuchungsgesamtheit einer dieser Parteien angehör(t)en, da der Auswahlwettbewerb für den höheren AD Personen mit extremen politischen Positionen bereits herausfiltert. Dennoch wurde die getroffene Annahme anhand der Werte auf einer zehnfach abgestuften politischen LinksRechtsskala (Fragen 15-16) überprüft. Der Durchschnittswert lag bei 4,74, wobei Extrempositionen nicht vertreten waren. 
able Jura gibt an, ob die Person Jura (1) oder eine andere Fachrichtung (0) studiert hat. Es wird jeweils nur das Hauptfach oder Erststudium betrachtet.

Mit Hypothese $4 \mathrm{a}$ untersuchen wir, ob die Chancen auf Berufserfolg im AD mit einer Tätigkeit in der Zentrale steigen. Diese Information lässt sich der Auflistung der Posten entnehmen. Neben den Dienstposten in den verschiedenen Abteilungen im AA wurden auch Verwendungen im Bundeskanzleramt, im Bundespräsidialamt, bei Parteien und in den Bundesländern (z.B. persönlicher Referent beim Ministerpräsident) als Jahre in der Zentrale kodiert. Die dichotome Variable Zentrale gibt an, ob die Person unter- (0) oder überdurchschnittlich (1) viel Zeit auf Dienstposten im Inland verbracht hat.

Hypothese $4 \mathrm{~b}$ besagt, dass vor allem der Aufenthalt in machtnahen Arbeitsbereichen im Inland einen positiven Einfluss auf den Berufserfolg hat. Die Variable Machtnähe gibt an, ob ein Diplomat während seiner Laufbahn im Bundeskanzleramt, im Bundespräsidialamt oder im Ministerbüro tätig war (1) oder nicht (0). ${ }^{16}$ Die Operationalisierung des Konstrukts Konformismus soll erfassen, ob die Interviewpartner ihre Einstellungen auf die offiziellen Haltungen der Regierungsparteien abstimmten oder nicht. Da die ,karriererelevanten Einstellungen" sich im AD hauptsächlich auf die Außenpolitik konzentrieren, braucht man Indikatoren, die die Anpassung an die Regierungsaußenpolitik erfassen. Im Untersuchungszeitraum (1970 bis 2008) fanden zwei grundlegende politische Ereignisse statt: der Wechsel von der sozialliberalen zur schwarz-gelben Koalition (1982) und von der schwarz-gelben zur rot-grünen Koalition (1998). ${ }^{17}$ Wenn eine Einstellungsanpassung stattgefunden hat, sollte sie vor allem bei diesen politischen Wechseln zum Tragen kommen. Es soll also weniger der Grad der Anpassung an die Regierungspolitik als an die Koalitionspolitik gemessen werden. Da sich die deutsche Außenpolitik seit dem zweiten Weltkrieg nicht nur durch Kontinuität, sondern auch durch einen weitgehenden überparteilichen Konsens auszeichnet (Haftendorn 2001; Overhaus u. Schieder 2002; Maull 2006; Risse 2004), lassen sich nur schwer inhaltliche Dimensionen identifizieren, auf denen die Parteien - geschweige denn die Koalitionen - grundlegend voneinander abgewichen sind. Ohne eine klare Dimensionierung erweist sich die Messung von Veränderungen bzw. Anpassungen der außenpolitischen Einstellungen uber die Zeit als schwierig. Hinzu kommt, dass Einstellungen in dieser Untersuchung nur ein Mal und ex post gemessen werden konnten.

16 Wir überprüften auch mit Dummyvariablen, ob sich die Arbeit in anderen machtnahen Arbeitsbereichen auszahlt (,Leitungsstab“, „Planungsstab“ und „persönlicher Referent der Staatssekretäre oder Staatsminister"). Solche Zusammenhänge ließen sich aber zum Teil wegen geringer Fallzahlen nicht nachweisen. Ähnlich gilt, dass nur vier Diplomaten während ihrer Laufbahn im Ministerbüro dienstlich tätig waren. Daher wurde der Indikator Ministerbüro mit dem Bundeskanzleramt-Dummy zur Variablen Machtnähe zusammengefasst.

17 Dreiunddreißig Prozent der Diplomaten waren im Jahr 2005, zu Beginn der Legislaturperiode der großen Koalition, bereits aus dem AD ausgeschieden. Weitere $46 \%$ sind bis 2008 ausgeschieden. Für diese war eine Beförderung im Rahmen der großen Koalition nicht mehr pensionsrelevant (BeamtVG § 5 IV). Da der Höhepunkt der Karriere den Aussagen der Interviewpartner zufolge zwischen 45 und 55 Jahren erreicht wird, ist zu vermuten, dass dieser Wechsel auch für die Karrieren der restlichen $21 \%$ nicht mehr von allzu großer Bedeutung war. Daher und aus Gründen der besseren Vergleichbarkeit wird die große Koalition in dieser Untersuchung nicht als karriererelevant betrachtet. 
Aus diesen Gründen greifen wir auf eine indirekte Messung des Konformismus zurück. So baten wir die Interviewpartner, die Außenpolitik der jeweiligen Koalitionen auf einer Likert-Skala von 0 (negativ) bis 10 (positiv) zu bewerten. ${ }^{18}$ Basierend auf diesen Items wurde ein Summenwert für die Gesamtbewertung der Außenpolitik über die drei Koalitionen hinweg erstellt. Es wurde angenommen, dass Konformisten eine höhere Gesamtbewertung abgeben als Prinzipientreue. ${ }^{19}$

Der Indikator wurde dichotomisiert, wobei 0 eine unterdurchschnittliche Bewertung und 1 eine überdurchschnittliche Bewertung angibt. Im Zusammenhang mit Hypothese 5 wird vermutet, dass eine überdurchschnittlich hohe Bewertung der Koalitionspolitik sich positiv auf den Berufserfolg auswirkt.

Kontrollvariablen: In dieser Untersuchung soll für zwei zusätzliche Faktoren kontrolliert werden, die sich in der bisherigen Literatur zum Karriereerfolg im öffentlichen Dienst als einflussreich erwiesen haben: die Promotion und die Berufsjahre. ${ }^{20}$ Dreher (1996, S. 69) stellt fest, dass die Promotion nicht nur einen ,präsentablen Pluspunkt" bei „engen" Selektionsentscheidungen beim Eintritt in den öffentlichen Dienst, sondern auch ein wichtiges Zugangskriterium für Spitzenpositionen darstellt. Dies ist damit zu begründen, dass „Personalverantwortliche davon ausgehen, dass die wissenschaftliche Herangehensweise Problemlösungsprozesse bei der Programmentwicklung erleichtert". Die zusätzliche „wissenschaftliche Ausgewiesenheit“ promovierter Diplomaten sollte ihnen vor allem im Rahmen der konzeptionellen Arbeit in der Zentrale zugute kommen. In der Empirie hat sich die Karrierewirksamkeit der Promotion in der öffentlichen Verwaltung weitestgehend bestätigt (v. Beyme 1971, S. 57; Steinkemper 1974, S. 119; Derlien 1994, S. 257). Die Promotion stellt in dieser Untersuchung das einzige Merkmal dar, welches als Leistungskriterium bezeichnet werden kann. ${ }^{21}$ Das Vorliegen des Merkmals ,promoviert" konnte dem Fragebogen entnommen werden und wurde mit 1 bzw. 0 gekennzeichnet.

18 Man könnte annehmen, dass bei einer allgemeinen Bewertung der Außenpolitik der Koalitionen indirekt eine Bewertung der gesamten Politik oder der Personalpolitik der Koalitionen erfolgt. Daher wurde nach der Bewertung der Koalitionspolitik aufgefächert in acht Außenpolitikbereiche gefragt. Eine Rangkorrelation von 0,76 zwischen der Konformismusskala und dieser Kontrollvariable bestätigt die Validität des Messinstrumentes für die Bewertung der Außenpolitik.

19 Es wurde weiterhin vermutet, dass ein hoher Summenwert mit einer niedrigen Varianz der Teilbewertungen einhergeht. Tatsächlich fällt eine überdurchschnittliche Bewertung der Koalitionspolitik bei $80 \%$ der Befragten mit einer unterdurchschnittlichen Varianz zusammen.

20 Ein weiterer Einflussfaktor, der seine Erklärungskraft in der Karrierenliteratur bewiesen hat, ist das Geschlecht (Luhmann u. Mayntz 1973, S. 140; Pippke 1975, S. 108, 153). Allerdings wird hier aufgrund des niedrigen Frauenanteils in der Untersuchungsgesamtheit (unter 7\%) nicht dafür kontrolliert. Die Tatsache, dass es bis zum Untersuchungszeitpunkt nur eine Diplomatin mit der Besoldungsgruppe B9 im AD gab, spricht jedoch für sich.

21 Dass hier keine weiteren Leistungskriterien untersucht werden, hat verschiedene Gründe: Zunächst nehmen wir an, dass Diplomaten aufgrund des strengen Auswahlwettbewerbs des $A D$ bereits überdurchschnittlich leistungsfähig sind. Innerdienstliche Leistungen, die - entsprechend des Leistungsgrundsatzes (Art $33 \mathrm{II} \mathrm{GG)} \mathrm{-} \mathrm{einen} \mathrm{starken} \mathrm{Einfluss} \mathrm{auf} \mathrm{den} \mathrm{Karriereerfolg}$ ausüben sollten, entziehen sich zudem weitgehend empirischer Messbarkeit (Pippke 1975, S. 44). 
Die Anciennität, also die Rangfolge aufgrund des Dienstalters, gilt als wichtiges formales Aufstiegskriterium im öffentlichen Dienst. So bemerkt Pippke (1975, S. 126), dass die Variable „Dienstalter" den „weitaus stärksten Einfluss auf die Besetzung von Spitzenpositionen"s sowie allgemein auf den Berufserfolg im öffentlichen Dienst hat. Es wird angenommen, dass mit steigendem Dienstalter auch die organisationsrelevanten Kenntnisse sowie Führungsqualitäten zunehmen. Das beamtenrechtliche Anciennitätsprinzip sowie der Erfahrungszuwachs bei steigendem Alter sollten dazu führen, dass ein Diplomat, der eine höhere Anzahl von Berufsjahren im AD absolviert, letztendlich auch eine höhere Karrierestufe erreichen kann. Die Berufsjahre ergeben sich aus der Postenaufl istung im Fragebogen.

\subsection{Statistisches Modell}

Ziel des statistischen Modells ist in dieser Untersuchung die Voraussage von Berufserfolg im höheren $\mathrm{AD}$. Da die abhängige Variable ordinalskaliert ist, entschieden wir uns wegen der einfacheren Interpretation für das ordinale Logit-Modell als Schätzansatz im Vergleich zum ordinalen Probit-Ansatz (McCullagh 1980, S. 116). Eine wichtige Annahme des Schätzansatzes ist die Parallel-regression- oder Proportional-odds-Annahme. Diese besagt, dass der Einfluss der unabhängigen Variablen über die Kategorien der abhängigen Variablen hinweg konstant ist. Dies ist dann der Fall, wenn die Regressionskoeffizienten bei binären Regressionen, in denen eine Kategorie auf der Ordinalskala den Wert 1 hat, der Rest der Ausprägungen den Wert 0, annähernd gleich sind bzw. deren Kurven parallel verlaufen. Die Parallel-regression-Annahme, die eine Voraussetzung für eine sinnvolle Interpretation des ordinalen Logit-Modells darstellt, wird oft nicht erfüllt. Um zu prüfen, ob diese Annahme verletzt wird, führen wir Approximate-Likelihood-Ratio-Tests durch (Long u. Freese 2006, S. 199).

Als Gütemaß wurde hier McKelveys und Zavoinas $\mathrm{R}^{2}$ gewählt, da nach Simulationen dies für ordinale abhängige Variablen die beste Annäherung an das $R^{2}$ der linearen Regression darstellt (Hagle u. Mitchell 1992; Windmeijer 1995). Des Weiteren werden wir das Adjusted Count $\mathbf{R}^{2}$ auffuhhren, um anzugeben, wie groß der Anteil der Beobachtungen ist, die das Modell im Vergleich zum Nullmodell korrekt klassifiziert.

Non-Response: Vor der Beschreibung des Datensatzes soll auf das Problem der ItemNonresponse im Fragebogen eingegangen werden. Es ergaben sich insgesamt vier fehlende Werte, die für die nachstehenden inferenzstatistischen Verfahren relevant sind. Diese vier Fälle haben sich auf die Variablen Bewertung der Außenpolitik und Parteimitgliedschaft verteilt. Da die entsprechenden Items als „sensibel“ eingestuft werden können, ist davon auszugehen, dass diese Werte nicht zufällig fehlen (im Sinne von ,missing not at random") (Rubin 1976; Schnell et al. 1999, S. 431). Aufgrund der geringen Fallzahl wurden diese vier Beobachtungen jedoch nicht aus der Analyse ausgeschlossen.

Im Falle der Parteimitgliedschaft wurden die fehlenden Werte aufgrund theoretischer Überlegungen ersetzt. Es sind zwei Angehörige der höchsten Besoldungsgruppe (B9) in der Stichprobe, die bei diesen Items keine Angabe gemacht haben. Es ist wahrscheinlich, dass dies auf ihre Stellung als hohe politische Beamte zurückzuführen ist. Eine der beiden Personen hat zwar keine Aussage zur Parteimitgliedschaft gemacht, gab jedoch an, über die Laufbahn hinweg mehreren Parteien zugeneigt zu haben. Bei der zweiten Person 
lässt ein Referentenposten im Inland auf eine Parteimitgliedschaft schließen. Die zweite Person wurde als Parteimitglied und die erste Person als Nicht-Mitglied kodiert. ${ }^{22}$ Bei den Variablen Bewertung der Außenpolitik fehlten ferner Angaben für zwei Beamte der Besoldungsgruppe B3. Da auch Drittvariablen kein theoretisch sinnvolles Ersetzen der fehlenden Werte ermöglichen, wurden sie in diesem Fall durch den Mittelwert ersetzt. ${ }^{23}$

\section{Analyse und Ergebnisse}

Diese Studie untersucht aufgrund von 51 Befragungen den beruflichen Erfolg im AD. ${ }^{24}$ Tabelle 1 zeigt die Lage- und Streuungsmaße der Untersuchungsvariablen einschließlich der ersetzten Werte. Die abhängige Variable unterteilt sich in die Kategorien 1 (A15B3), 2 (B6) und 3 (B9). Der Großteil der Diplomaten gehört der untersten Kategorie der abhängigen Variablen an. Die Verteilung der abhängigen Variablen ist rechtsschief, was dem pyramidenförmigen Stellenkegel im AD entspricht.

Mit Ausnahme der kontinuierlichen Variablen Berufsjahre sind alle unabhängigen Variablen dichotom. Die vermutete Einflussrichtung der Kovariaten auf den Karriereerfolg ist durchweg positiv. Vierzehn Prozent der Probanden sind Amtskinder. Die Hälfte der Befragten hat angegeben, Parteimitglied zu sein. Davon neigen $32 \%$ entweder keiner oder mehreren Parteien zu; $40 \%$ neigen der SPD, 16\% der FDP und $8 \%$ der CDU/ CSU zu. ${ }^{25}$ Die Variable Parteineigung zeigt, dass sich ein Großteil der Parteimitglieder auf die SPD und FDP verteilt. Dies liegt möglicherweise daran, dass die Probanden zur Zeit der sozialliberalen Koalition in den AD eingetreten sind. ${ }^{26}$ Circa $55 \%$ der Probanden haben ein juristisches Erststudium absolviert. Wenn man diesen Anteil mit dem in der Untersuchungsgesamtheit vergleicht $(65 \%)$, wird deutlich, dass die Juristen in der Stichprobe unterrepräsentiert sind. Durchschnittlich haben die Diplomaten etwas mehr

22 Aufgrund der Verteilung der Variablen Parteimitgliedschaft in der gesamten Stichprobe und insbesondere in der Gruppe der B9er (in beiden Fällen $50 \%$ ) erschien dies aus methodischer Sicht vertretbar.

23 Da ein Ersetzen von fehlenden Werten zu einer Verzerrung der Ergebnisse bzw. zu einer Unterschätzung der Varianz in der Stichprobe führen kann, wurden die Modelle zur Kontrolle mit allen möglichen Kombinationen von ersetzten Werten für die drei Variablen gerechnet. Es ergaben sich keine wesentlichen Unterschiede in den Ergebnissen.

24 Abgesehen von den Daten aus den Fragebögen wurden über das Schneeballverfahren Adresslisten für die vier untersuchten Diplomatenlehrgänge ermittelt. Diese enthalten neben den Kontaktdaten auch die Studienrichtung, das Geburtsjahr und die letzte oder derzeitige dienstliche Verwendung der Diplomaten. Des Weiteren konnten über die Webseiten der Auslandsvertretungen 50 Lebensläufe eingesehen werden. Die entsprechenden Daten wurden gemäß Codeplan (siehe Online-Anhang) in den Datensatz übertragen. Anhand dieser zusätzlichen Daten können Aussagen über die Repräsentativität der Stichprobe angestellt werden.

25 Die fehlenden Prozente entsprechen Probanden, die die Frage nach einer Parteimitgliedschaft positiv beantwortet, aber keine Angabe zur Parteineigung gemacht haben.

26 Dies kann als Hinweis darauf gesehen werden, dass die politische Gesinnung für viele ausschlaggebend für die Bewerbung beim $\mathrm{AD}$ war und dass sich diese im Untersuchungszeitraum beim Großteil der Probanden nicht wesentlich verändert hat. 
Tab. 1: Deskriptive Statistik für die untersuchten Variablen

\begin{tabular}{llllll}
\hline Abhängige Variable & $\mathrm{N}$ & Modus & Erstes Quartil & Median & Drittes Quartil \\
\hline Berufserfolg & 51 & 1 & 1 & 1 & 2 \\
\hline Unabhängige Variablen & $\mathrm{N}$ & Mittelwert & Standardabweichung & Minimum & Maximum \\
\hline Amtsbonus & 51 & 0,14 & 0,35 & Dummy-Variable \\
Parteimitgliedschaft & 51 & 0,49 & 0,50 & Dummy-Variable \\
Jura & 51 & 0,55 & 0,50 & Dummy-Variable \\
Jahre in der Zentrale & 51 & 0,51 & 0,50 & Dummy-Variable \\
(Mittelwert-Variable) & & & & \\
Machtnähe & $50^{\mathrm{a}}$ & 0,16 & 0,37 & Dummy-Variable \\
Bewertung d. & 51 & 0,57 & 0,49 & Dummy-Variable \\
Koalitionspolitik & & & & \\
(Mittelwert-Variable) & & & & Dummy-Variable \\
Promotion & 51 & 0,57 & 0,50 & 29 \\
Berufsjahre & 51 & 32,86 & 1,82 & 37 \\
\hline
\end{tabular}

aine Person hat bei einer Frage nur ihre Verwendungen im Ausland angegeben. Daher das $\mathbf{n}$ von 50

als zwölf Jahre auf Posten im Inland verbracht, was tatsächlich in etwa einem Drittel der Berufsjahre entspricht (vgl. Abschn.2). Insgesamt waren etwa 16\% der Probanden mindestens einmal dienstlich im Bundeskanzleramt, Bundespräsidialamt oder Ministerburo tătig (Variable: Machtnähe). Siebenundfünfzig Prozent der Diplomaten haben die Außenpolitik der verschiedenen Koalitionen überdurchschnittlich positiv beurteilt. Promovierte machen $57 \%$ der Stichprobe aus. ${ }^{27}$

\subsection{Multivariate Analyse}

Nachdem ein erster Überblick über den Datensatz gegeben wurde, sollen nun die Ergebnisse der ordinalen logistischen Regression vorgestellt werden. Die ersten zwei Spalten der Tab. 2 zeigen die Sozialisationsmodelle (Soz 1 und Soz 2), während in den zwei letzteren die Rational-Choice-Modelle (RC 1 und RC 2) aufgeführt sind.

Das erste Sozialisationsmodell umfasst neben den Kontrollvariablen die Prädiktoren Amtsbonus und Parteimitgliedschaft. Beide Variablen erreichen jedoch keine statistische Signifikanz. Mit einem Adjusted Count $\mathrm{R}^{2}$ von $5 \%$ ist dieses erste Modell dem Nullmodell in seiner Erklärungskraft nicht wesentlich überlegen. Diesen Eindruck bestätigt der nicht-signifikante $\mathrm{Chi}^{2}$-Wert. ${ }^{28}$ Ähnlich wenig aussagekräftig ist das zweite Sozialisationsmodell, das auch die Hybrid-Variable Jura umfasst. Obwohl das Vorzeichen des Koeffizienten wie vermutet positiv ist, tritt auch hier kein signifikanter Zusammenhang

27 Den Adresslisten zufolge beläuft sich der Anteil an Promovierten in den vier Diplomatenlehrgängen auf $47 \%$. Promovierte sind, im Gegensatz zu Juristen, in der Stichprobe überrepräsentiert. Dies muss bei der Evaluation der Ergebnisse in Betracht gezogen werden.

28 Die Wald-Statistik (Wald $\mathrm{Chi}^{2}$ ) und der entsprechende $\mathrm{p}$-Wert geben an, ob die Nullhypothese, derzufolge der Effekt aller Kovariaten gleichzeitig dem Wert Null entspricht, verworfen werden kann. In diesem Fall kann die Nullhypothese nicht verworfen werden. 
Tab. 2: Sozialisations- und Rational-Choice-Modelle

\begin{tabular}{|c|c|c|c|c|}
\hline Variablen & Soz 1 & Soz 2 & $\mathrm{RC} 1$ & $\mathrm{RC} 2$ \\
\hline \multirow[t]{2}{*}{ Amtsbonus } & $-0,92$ & $-0,91$ & & \\
\hline & $(0,94)$ & $(0,94)$ & & \\
\hline \multirow[t]{2}{*}{ Parteimitgliedschaft } & $-0,01$ & $-0,00$ & & \\
\hline & $(0,58)$ & $(0,58)$ & & \\
\hline \multirow[t]{2}{*}{ Jura } & & 0,34 & 0,77 & \\
\hline & & $(0,61)$ & $(0,81)$ & \\
\hline \multirow[t]{2}{*}{ Zentrale } & & & $2,56^{* * *}$ & $2,35^{* * *}$ \\
\hline & & & $(0,81)$ & $(0,76)$ \\
\hline \multirow[t]{2}{*}{ Machtnähe } & & & $2,75^{* * *}$ & $2,94^{* * *}$ \\
\hline & & & $(1,01)$ & $(1,00)$ \\
\hline \multirow[t]{2}{*}{ Bewertung d. Koalitionspolitik } & & & $1,51^{* *}$ & $1,51^{* *}$ \\
\hline & & & $(0,75)$ & $(0,75)$ \\
\hline \multirow[t]{2}{*}{ Promotion } & 0,84 & 0,84 & $1,68^{*}$ & $1,61^{*}$ \\
\hline & $(0,61)$ & $(0,62)$ & $(0,86)$ & $(0,84)$ \\
\hline \multirow[t]{2}{*}{ Berufsjahre } & $0,32^{*}$ & 0,28 & $0,44^{*}$ & $0,49^{* *}$ \\
\hline & $(0,17)$ & $(0,18)$ & $(0,25)$ & $(0,24)$ \\
\hline \multirow[t]{2}{*}{ Schwellenwert I } & $11,11^{*}$ & $10,18^{*}$ & $18,81^{* *}$ & $19,95^{* *}$ \\
\hline & $(5,76)$ & $(5,99)$ & $(8,54)$ & $(8,26)$ \\
\hline \multirow[t]{2}{*}{ Schwellenwert 2} & $12,97^{* *}$ & $12,04^{* *}$ & $21,76^{* *}$ & $22,81^{* * *}$ \\
\hline & $(5,82)$ & $(6,04)$ & $(8,72)$ & $(8,45)$ \\
\hline Wald $\mathrm{Chi}^{2}$ & 4,83 & 5,14 & $18,34^{* * *}$ & $18,55^{* * *}$ \\
\hline McKelveys und Zavoinas $\mathrm{R}^{2}$ & 0,15 & 0,16 & 0,61 & 0,58 \\
\hline $\mathrm{BIC}$ & $-87,35$ & $-83,73$ & $-102,85$ & $-105,86$ \\
\hline Adj. Count $\mathrm{R}^{2}$ & 0,05 & 0,05 & 0,48 & 0,43 \\
\hline Log Likelihood & $-44,79$ & $-44,63$ & $-30,73$ & $-31,18$ \\
\hline $\mathrm{N}$ & 51 & 51 & 50 & 50 \\
\hline
\end{tabular}

Die Schwellenwerte sind Schätzparameter des ordinalen logitischen Modells, die Schnittpunkte einer latenten Variablen für den Kategorienwechsel angeben. Sie dienen der Vorhersage von Wahrscheinlichkeiten für einzelne Beobachtungen und werden im Normalfall nicht inhaltlich interpretiert (s. Long u. Freese 2006)

${ }^{* * *} \mathrm{p}<0,01 ;{ }^{* *} \mathrm{p}<0,05 ;{ }^{*} \mathrm{p}<0,1$

hervor. Bei den Koeffizienten der anderen Kovariaten sowie bei den verschiedenen Gütemaßen zeigen sich keine wesentlichen Unterschiede im Vergleich zur ersten multivariaten Regression.

Insgesamt weisen also die Sozialisationsvariablen nur geringe Erklärungskraft in Bezug auf den Karriereerfolg von Diplomaten auf. Amtskinder, von denen man erwartet hätte, dass sie aufgrund einschlägiger Sozialisation bessere Karriereaussichten haben als ihre Kollegen, schneiden im Schnitt sogar schlechter ab. Die Parteimitgliedschaft und die damit einhergehende langjährige Identifizierung mit einer der Regierungsparteien bleiben weitgehend ohne Einfluss im Hinblick auf den Karriereerfolg. Auch das Studium der Rechtswissenschaften scheint keine signifikante Erfolgswirksamkeit zu besitzen. 
Tab. 3: Ergebnisse des Modells RC 2 als Relatives Risiko (Odds Ratios)

\begin{tabular}{lclc}
\hline Berufserfolg & Koeffizienten & $\begin{array}{l}\text { Relatives Risiko } \\
\text { (Odds Ratio) }\end{array}$ & $\begin{array}{l}\text { Prozentuale Veränderung } \\
\text { im rel. Risiko }\end{array}$ \\
\hline Zentrale & $2,35^{* * *}$ & 10,43 & 942,9 \\
Machtnähe & $\begin{array}{c}(0,76) \\
2,94^{* * *}\end{array}$ & 18,99 & 1799,2 \\
& $\begin{array}{c}(1,00) \\
1,51^{* *}\end{array}$ & 4,52 & 352,0 \\
Bewertung d. Koalitionspolitik & $(0,75)$ & & \\
& $1,61^{*}$ & 4,99 & 398,9 \\
Promotion & $(0,84)$ & & 63,2 \\
Berufsjahre & $0,49^{* *}$ & 1,63 & 50 \\
& $(0,24)$ & & \\
$\mathrm{N}$ & 50 & 50 & \\
${ }^{* * *} \mathrm{p}<0,01 ;{ }^{* *} \mathrm{p}<0,05 ;{ }^{*} \mathrm{p}<0,1$ & & &
\end{tabular}

Anders verhält es sich bei den Rational-Choice-Modellen, die in der dritten und vierten Spalte aufgeführt sind. Im ersten rationalistischen Modell (RC 1) wird der Einfluss der Rational-Choice-Variablen zusammen mit den Kontrollvariablen getestet. Auch hier wurde der Vollständigkeit halber die Hybrid-Variable Jura miteinbezogen, die weiterhin ohne signifikanten Einfluss bleibt. ${ }^{29}$ Wenn man die Variable Jura weglässt, erhält man Modell RC 2, welches als sparsameres Modell die Grundlage für die Interpretation der Ergebnisse unserer Untersuchung darstellt.

Mit einem Pseudo $\mathrm{R}^{2}$ von 0,58 weist Modell $\mathrm{RC} 2$ einen eindeutigen Vorsprung gegenüber den Sozialisationsmodellen auf. Insgesamt werden $76 \%$ der Diplomaten der richtigen Besoldungsgruppe zugeordnet. ${ }^{30}$

In Tab. 3 sind neben den Regressionskoeffizienten, das relative Risiko in Form der Odds Ratios und die prozentualen Veränderung im relativen Risiko für das Modell RC 2 aufgeführt. Der stärkste positive Einfluss geht von der Variablen Machtnähe aus: Für eine Person, die während ihrer Amtszeit im Bundeskanzleramt, Bundespräsidialamt oder Ministerbüro arbeitet, ist die Chance auf Berufserfolg im AD etwa 19 Mal so hoch wie für andere Diplomaten, wenn alle weiteren Variablen konstant gehalten werden. ${ }^{31}$ Der signifikant positive Einfluss der Variablen Zentrale zeigt jedoch auch, dass eine überdurch-

29 Dies bestätigt auch der Wald Test: Wald $\mathrm{Chi}^{2}(1)=0,88 ; \mathrm{p}>\mathrm{Chi}^{2}=0,35$.

30 In Modell RC 1 beläuft sich dieser Anteil auf 78\% und in den Sozialisationsmodellen jeweils auf $59 \%$.

31 Allerdings stellt sich bei der Variablen Machtnähe das Problem der Kausalrichtung: Führt die dienstliche Verwendung im machtnahen Arbeitsbereich zum Berufserfolg, oder führt der Berufserfolg in den Arbeitsbereich? Einen Hinweis darauf kann der Zeitpunkt geben, zu welchem die Diplomaten während ihrer Laufbahn im Ministerbüro, Bundeskanzleramt oder Bundespräsidialamt tätig waren. Bei genauerer Betrachtung zeigt sich, dass kein systematischer Zusammenhang zwischen dem Zeitraum, in dem die Diplomaten in dem $/ \mathrm{n}$ machtnahen Arbeitsbereich/en waren, und der erreichten Besoldungsgruppe besteht. Diese Positionen scheinen demnach nicht nur den ,erfolgreichen Diplomaten" vorbehalten zu sein. 
schnittliche Anzahl von Berufsjahren im Inland sich - unabhängig von den jeweiligen Arbeitsbereichen - positiv auf den Berufserfolg auswirkt. Ein Diplomat, der mehr als das übliche Drittel seiner Berufszeit in der Zentrale verbringt, hat circa zehn Mal höhere Erfolgschancen als jener, der überdurchschnittlich viel Zeit auf Auslandsposten verbringt. Der Indikator für Konformismus erreicht in Modell RC 2 ein Signifikanzniveau von $5 \%$. Eine überdurchschnittliche Bewertung der Koalitionspolitik erhöht demzufolge die Erfolgschancen um 352\%.

Die zwei Kontrollvariablen erreichen ebenfalls statistische Signifikanz. Ausgehend von den vorliegenden Daten erhöht eine Promotion die Wahrscheinlichkeit für Berufserfolg fast um $400 \%$. Auch im Hinblick auf die Variable Berufsjahre sind die Ergebnisse konsistent mit der bisherigen Forschung zu Karrieren im öffentlichen Dienst: Mit jedem zusätzlichen Berufsjahr steigt die Erfolgswahrscheinlichkeit um 63\%, wenn man die anderen Prädiktoren konstant hält.

Der Vergleich der Modelle in Tab. 2 zeigt, dass die Rational-Choice-Variablen eine wesentlich höhere Erklärungskraft in Bezug auf den Karriereerfolg von Diplomaten aufweisen als die Sozialisationsvariablen. Die multivariate Analyse führt somit zur Ablehnung der Sozialisationshypothesen sowie der Hybrid-Hypothese zugunsten der Rational-Choice-Hypothesen.

Die Regressionsdiagnostik sowie eine genauere Analyse der Daten verleitet jedoch zu einer gewissen Vorsicht. Die Parallel-Regression-Annahme, die als Voraussetzung für eine sinnvolle Interpretation der Ergebnisse gilt, wird sowohl im zweiten Sozialisationswie im ersten RC-Modell verletzt. ${ }^{32}$ Bei näherer Betrachtung stellt sich vor allem die Vari-able Jura als problematisch heraus. Dies bedeutet, dass die entsprechenden Koeffizienten je nach Kategorie der abhängigen Variablen variieren. Kreuztabellen zeigen, dass Juristen und Nicht-Juristen in den unteren beiden Kategorien der abhängigen Variablen (A15-B6) zwar etwa gleichverteilt sind, dass in der obersten Kategorie beruflichen Erfolgs jedoch alle Diplomaten dieser Stichprobe ein juristisches Studium absolviert haben. ${ }^{33}$ Obwohl Hypothese 3 im Rahmen der multivariaten Analyse keine Bestätigung findet, spricht die Verteilung der Variablen Jura eindeutig dafür, dass Juristen höhere Erfolgschancen im AD haben. Das Jurastudium zeigt zwar keinen differenzierenden Einfluss bei den Besoldungsgruppen bis B6, scheint aber für die untersuchten Kohorten ein wichtiges Aufstiegskriterium im Hinblick auf die Spitzenpositionen darzustellen.

Hypothese 2, derzufolge die Parteimitgliedschaft in einer der Regierungsparteien einen positiven Einfluss auf die Karriere im $\mathrm{AD}$ hat, findet keine Bestätigung im Rahmen der multivariaten Analyse. Um für Multikollinearität zu kontrollieren, wurde eine bivariate Korrelationsmatrix aufgestellt. Dabei ist aufgefallen, dass die Variablen Parteimit-

32 Ergebnisse der Approximate-Likelihood-Ratio-Tests: Modell Soz 2: Chi ${ }^{2} \quad(4)=23,57$, $\mathrm{p}>\mathrm{Chi}^{2}=0,00$; Modell RC 1: $\mathrm{Chi}^{2}(6)=18,48, \mathrm{p}>\mathrm{Chi}^{2}=0,01$.

33 Bei der Variablen Jura liegen Daten für die gesamte Grundgesamtheit $(N=133)$ vor. Bei Betrachtung der bivariaten Zusammenhänge für die 112 Beobachtungen, für die auch die Besoldungsgruppe bekannt ist, mildert sich das oben gezeichnete Bild nur bedingt: Von 16 Angehörigen der Besoldungsgruppen B9-B11 haben 14 Jura studiert. 
gliedschaft und Machtnähe signifikant korrelieren. ${ }^{34}$ Von zehn Diplomaten, die während ihrer Laufbahn im Bundeskanzleramt, Bundespräsidialamt oder Ministerbüro tätig waren, sind sieben Parteimitglieder der SPD oder FDP. Fünf von ihnen waren unter ihrer Partei in einem der machtnahen Arbeitsbereiche tätig. Die „Mächtigen“ (Außenminister und Bundeskanzler) scheinen bevorzugt Mitglieder ihrer Partei als direkte Untergebene auszuwählen. Obwohl eine Parteimitgliedschaft keinen direkten Einfluss auf den Berufserfolg zu haben scheint, kann sie wohl Türen öffnen und eine wesentliche Voraussetzung dafür sein, dass ein Diplomat zunächst in den Dunstkreis der Macht gerät, um dann in der Hierarchie auch formal aufzusteigen. Um den Einfluss der Parteimitgliedschaft vollständig evaluieren zu können, benötigt man allerdings eine größere Stichprobe, bei der nach Parteibuch differenziert werden kann.

In diesem Zusammenhang soll herausgestellt werden, dass die Studie nur Repräsentativität für die untersuchten Kohorten beanspruchen kann. Für die Untersuchungsgesamtheit konnten gewisse Zusammenhänge, die keiner Fragebogendaten bedurften, auch für eine höhere Fallzahl überprüft werden. Dabei bestätigen sich die Einflüsse der erhobenen biografischen Merkmale (Studienfach, Zentrale, Machtnähe, Berufsjahre). Einzig der Faktor Promotion verliert in diesem ausgeweiteten Sample seine statistische Signifikanz.

Rein soziodemografisch gesehen unterscheiden sich die heutigen AD-Crews von den hier untersuchten, wie ein Vergleich unserer Daten mit jenen von drei jüngeren Jahrgängen ergibt. So ist der Frauenanteil (41\%) heute z. B. deutlich höher, der Anteil von Juristen $(25 \%)$ und Promovierten (16\%) hingegen sehr viel niedriger..$^{35}$

Solche Veränderungen an der Rekrutierungsbasis zeigen aber noch nicht schlüssig, dass sich auch die Karrieredeterminanten, wie wir sie hier untersucht haben, grundlegend gewandelt hätten. So hat sich der positive Einfluss von Konformismus auf die Diplomatenkarriere bestätigt. Die entsprechende rationalistische Hypothese kann allerdings nur unter Vorbehalt angenommen werden. Erstens ist die Validität des Messinstruments infrage zu stellen. Um eine Einstellungsveränderung zu messen, benötigt man ein Längsschnittdesign mit verschiedenen Messzeitpunkten. Da die Probanden in diesem Fall retrospektiv zu ihrer Meinung befragt wurden, ist nicht auszuschließen, dass eine nachträgliche Rationalisierung ihre Antworten verzerrt (Schnell et al. 1999, S. 314). Zweitens lässt sich im Rahmen eines Querschnittdesigns die Richtung der Kausalität nicht eindeutig bestimmen. Die Frage, ob die positive Einstellung zum Dienstherrn zu Karriereerfolg führt oder umgekehrt, bleibt offen. Dennoch ist die nachgewiesene Korrelation zwischen konformistischen Einstellungen und Berufserfolg ein Indiz dafür, dass auch die moderne Verwaltung sich noch immer schwer tut mit Beamten, die gegenüber der offiziellen Politik Vorbehalte haben.

34 Wenn man zwei weitere Beobachtungen, für die die Parteimitgliedschaft dem Lebenslauf entnommen werden konnte, hinzuzieht, ergibt sich ein Korrelationskoeffizient von 0,30, welcher auf dem 5 Prozent-Niveau signifikant ist.

35 Diese Daten beschreiben die Attaché-Lehrgänge der Jahre 2007, 2008 und $2009(n=123)$. Die entsprechenden Angaben wurden auf der Homepage des AA veröffentlicht: http://www.auswaertiges-amt.de/diplo/de/AAmt/AusbildungKarriere/AA-Taetigkeit/HoehererDienst/Uebersicht. html (Zugriff am 21.04.2008 und 21.11.2009). 


\section{Schlussbetrachtung und Ausblick}

In dieser Untersuchung wurde der Frage nachgegangen, welche Karrierefaktoren im höheren AD maßgebend sind. Theoretischer Ausgangspunkt sind zwei prominente Ansätze aus den Sozialwissenschaften: der Sozialisations- und der Rational-Choice-Ansatz. Um festzustellen, welcher dieser Ansätze die höhere Erklärungskraft in Bezug auf den Karriereerfolg von Diplomaten aufweist, wurden sechs Hypothesen aufgestellt, die in Form von Sozialisations- und Rational-Choice-Hypothesen je einem der Ansätze zugeordnet wurden.

Auf der Basis von Daten aus einer schriftlichen Befragung, an der sich 51 Diplomaten des höheren AD beteiligt haben, kann man schließen, dass dem rationalistischen Modell die höhere Erklärungskraft zukommt. Hierbei tritt vor allem die strategische Postenwahl in den Vordergrund. Jemand, der sich dafür einsetzt, überdurchschnittlich viel Zeit in der Zentrale - und besonders im direkten Blickfeld der „Mächtigen“ - zu verbringen, hat höhere Erfolgschancen. Die „richtige“ Parteimitgliedschaft scheint sich indirekt auf den Karriereerfolg auszuwirken, indem sie die Chancen auf eine Verwendung in einem machtnahen Arbeitsbereich erhöht. Auch Konformisten, die sich der wechselnden Regierungspolitik anpassen, scheinen höhere Erfolgschancen im AD zu haben. Obwohl sich das Jurastudium im Rahmen der multivariaten Analyse nicht als einflussreich bewiesen hat, sind die höchsten Positionen im AD faktisch nach wie vor Juristen vorbehalten. Des Weiteren steigern eine Promotion sowie eine hohe Anzahl an Berufsjahren die Erfolgwahrscheinlichkeit.

Sozialisationsfaktoren, wie der Amtsbonus und die Mitgliedschaft in einer der Regierungsparteien, haben keinen direkten positiven Einfluss auf den Berufserfolg im AD. Es ist anzunehmen, dass sich derartige gruppenspezifische Merkmale vornehmlich auf die Rekrutierung in den höheren AD auswirken. Dies würde die überdurchschnittlich hohen Anteile an dieser Gruppe an Parteimitgliedern (50\%), Amtskindern (13\%) und auch Adligen (9\%) unter den rekrutierten Diplomaten erklären.

Die Repräsentativität und zeitliche Stabilität dieser Untersuchungsergebnisse ist zu relativieren. Die Ergebnisse müssten im Rahmen einer umfassenden Studie mit höherer Fallzahl überprüft werden. Eine solche Studie sollte Diplomatenlehrgänge verschiedener Eintrittsjahre berücksichtigen, um mögliche zeitliche Trends zu erfassen. In diesem Fall könnte der Karriereerfolg durch die Beförderungsgeschwindigkeit operationalisiert werden, die bewiesenermaßen eng mit der pensionsrelevanten Besoldungsgruppe zusammenhängt (vgl. Pippke 1975, S. 174; Dreher 1996, S. 640).

Insgesamt legt diese Untersuchung einen Grundstein für vergleichende Studien zum Personal diplomatischer Dienste. ${ }^{36}$ Darüber hinaus bietet sie Ansätze zur Untersuchung von Karrieren anderer Verwaltungseliten. Letztendlich ist nur eine der Variablen, nämlich

36 Vergleichende Studien diplomatischer Dienste gewinnen vor allem im Hinblick auf die Schaffung des europäischen Auswärtigen Diensts (Art. 13a III Vertrag von Lissabon) an Aktualität, zumal sich dieser nicht nur aus Beamten des Generalsekretariats des Europäischen Rats und der Kommission, sondern auch aus Karrierebeamten der nationalen Dienste zusammensetzt. Wenn das europäische diplomatische Corps unerforscht bleibt, könnte sich in wenigen Jahren die Frage stellen, wer die unbekannte Elite ist, die die Europäische Union nach außen vertritt. 
der Anteil der im Ausland verbrachten Dienstzeit, ein spezifisches Merkmal des diplomatischen Dienstes.

Die systematische Untersuchung der Karrierefaktoren im öffentlichen Dienst durch Außenstehende liefert Hinweise zur Verwirklichung des Leistungsgrundsatzes bzw. zum Gewicht extrafunktionaler Aufstiegskriterien (Offe 1970, S. 89). Studien wie die hier präsentierte können dem Dienstherrn somit als Korrektiv dienen und einen Beitrag zu mehr Transparenz in der Beförderungspraxis leisten.

Danksagung: Die Verfasser dieses Artikels möchten sich bei den zwei anonymen Gutachtern für die informativen Gutachten und bei der PVS-Redaktion bedanken. Die Replikationsdaten, das Codebook und weitere Materialien zu diesem Artikel stehen auf der Replikationsseite des zweiten Autors: http://www.polver.uni-konstanz.de/gschneider/arbeitspapiere/replikationsdaten/

\section{Literatur}

Apel, Helmut. 1993. Bildungshandeln im soziokulturellen Kontext: Studienfachwahl und Studiengestaltung unter dem Einfluß familialer Ressourcen. Wiesbaden: Deutscher Universitäts-Verlag.

Aspinwall, Marc D., und Gerald Schneider. 2000. Same menu, separate tables. European Journal of Political Research 38:1-36.

Beste, Ralf. 2007. Kanzleramt bremst Steinmeier aus. DER SPIEGEL, 28. Apr. 2007. http://www. spiegel.de/politik/deutschland/0,1518,480008,00.html. Zugegriffen: 3. Feb. 2008.

Beyme, Klaus von. 1971. Die politische Elite in der Bundesrepublik Deutschland. München: Piper.

Biehler, Gernot. 2000. Ämterpatronage im diplomatischen Dienst? Neue juristische Wochenschrift 53:2400-2402.

Boudon, Raymond. 1998. Limitations of rational choice theory. The American Journal of Sociology 104:817-828.

Brandt, Enrico, und Christian Buck. 2003. Auswärtiges Amt: Diplomatie als Beruf. 3. aktualisierte Aufl. Opladen: Leske \& Budrich.

Bundesministerium des Innern (BMI). Hrsg. 1984. Öffentlicher Dienst A-Z: Wichtige Begriffe des öffentlichen Dienstrechts in Stichworten. Stuttgart: Kohlhammer.

Bürklin, Wilhelm P., und Hilke Rebenstorf. 1997. Eliten in Deutschland: Rekrutierung und Integration. Opladen: Leske \& Budrich.

Checkel, Jeffrey T. 2005. International institutions and socialization in Europe: Introduction and framework. International Organization 59:801-826.

Chong, Dennis. 2000. Rational lives: Norms and values in politics and society. Chicago: University of Chicago Press.

Chow, Irene Hau-Siu. 2002. Organizational socialization and career success of Asian managers. International Journal of Human Resource Management 13:720-737.

Curtius, Klaus. 1974. Die Nachwuchsausbildung für den höheren Auswärtigen Dienst der Bundesrepublik Deutschland und der Vereinigten Staaten von Amerika. Frankfurt a.M.: Metzner.

Derlien, Hans Ulrich. 1988. Repercussions of government change on the career civil service in West Germany: The cases of 1969 and 1982. Governance 1:50-78.

Derlien, Hans Ulrich. 1994. Karrieren, Tätigkeitsprofil und Rollenverständnis der Spitzenbeamten des Bundes - Konstanz und Wandel. Verwaltung und Fortbildung 22:255-274.

Derlein, Hans Ulrich. 2003. Mandarins or managers? The bureaucratic elite in Bonn, 1970 to 1987 and beyond. Governance 16:401-428. 
Derlien, Hans Ulrich, und Gerhard Pippig. 1990. Die administrative Elite: Kontinuität und Wandel 1949 bis 1984. Der Bürger im Staat 40:32-35.

Dreher, Christiane. 1996. Karrieren in der Bundesverwaltung. Voraussetzungen, Merkmale und Etappen von Aufstiegsprozessen im öffentlichen Dienst. Berlin: Duncker \& Humblot.

Elster, Jon. 1989. Salomonic judgements: Studies in the limitations of rationality. Cambridge: Cambridge University Press.

End, Heinrich. 1969. Erneuerung der Diplomatie. Der Auswärtige Dienst der Bundesrepublik Deutschland, Fossil oder Instrument? Neuwied: Luchterhand.

Enke, Edo. 1974. Oberschicht und politisches System in der Bundesrepublik Deutschland. Soziale Mobilität und Karrieremuster von 800 Inhabern von Spitzenpositionen der westdeutschen Gesellschaft. Europäische Hochschulschriften, Reihe 22, Bd. 9. Frankfurt a.M.: Lang.

Fearon, James, und Alexander Wendt. 2002. Rationalism v. constructivism: A skeptical view. In Handbook of international relations, Hrsg. Walter Carlsnaes, Thomas Risse, und Beth A. Simmons, 52-72. London: Sage.

Ferejohn, John. 1991. Rationality and interpretation: Parliamentary elections in early stuart England. In The economic approach to politics, Hrsg. Kirsten Monroe, 154-185. New York: Harper Collins.

Haftendorn, Helga. 1978. Verwaltete Außenpolitik: Sicherheits- und entspannungspolitische Entscheidungsprozesse in Bonn. Köln: Wissenschaft und Politik.

Haftendorn, Helga. 2001. Deutsche Außenpolitik zwischen Selbstbeschränkung und Selbstbehauptung: 1945-2000. München: Deutsche Verlags-Anstalt.

Hagle, Timothy M., und Glenn E. Mitchell. 1992. Goodness-of-fit measures for probit and logit. American Journal of Political Science 36:762-784.

Hall, Douglas T. 1987. Careers and socialization. Journal of Management 13:301-321.

Heublein, Ulrich, und Dieter Sommer. 2002. Studienanfänger 2000/2001. Fachinteresse und berufliche Möglichkeiten bestimmen die Studienfachwahl. HIS-Kurzinformationen A2.

Hoffmann-Lange, Ursula. 1990a. Eliten in der modernen Demokratie. Fragestellungen, theoretische Ansätze und Ergebnisse der Eliteforschung. In Eliten in der Bundesrepublik Deutschland, Hrsg. Ursula Hoffmann-Lange und Hans Georg Wehling, 11-27. Stuttgart: Kohlhammer.

Hoffmann-Lange, Ursula. 1990b. Wer gehört zur Machtelite der Bundesrepublik? In Eliten in der Bundesrepublik Deutschland, Hrsg. Ursula Hoffmann-Lange und Hans Georg Wehling, 164-178. Stuttgart: Kohlhammer.

Hooghe, Liesbeth. 2001. The European Commission and the integration of Europe. Cambridge: Cambridge University Press.

Hooghe, Liesbeth. 2005. Several roads lead to international norms, but few via international socialization: A case study of the European Commission. International Organization 59:861-898.

Hurrelmann, Klaus. 2006. Einführung in die Sozialisationstheorie. 9. Aufl. Basel: Beltz.

Johnston, Alistair I. 2001. Treating international institutions as social environments. International Studies Quarterly 45:487-515.

Johnston, Alistair I. 2005. Conclusions and extensions: Toward mid-range theorizing and beyond Europe. International Organization 59:1013-1044.

Judge, Timothy A., Daniel M. Cable, John W. Boudreau, und Robert D. Bretz. 1995. An empirical investigation of the predictors of executive career success. Personnel Psychology 483:485-519.

Jupille, Joseph, James A. Caporaso, und Jeffrey T. Checkel. 2003. Integrating institutions: Rationalism, constructivism, and the study of the European Union. Comparative Political Studies 36:7-40.

Kato, Junko. 1996. Institutions and rationality in politics - Three variations of neo-institutionalism. British Journal of Political Science 26:553-582.

London, Manuel, und Steven A. Stumpf. 1982. Managing careers. Reading: Addison-Wesley. 
Long, J. Scott, und Jeremy Freese. 2006. Regression models for categorical dependent variables using stata. College Station: Stata Press.

Luhmann, Niklas, und Renate Mayntz. 1973. Personal im öffentlichen Dienst: Eintritt und Karrieren: Personaluntersuchung. Baden-Baden: Nomos.

Maanen, John. E. van, und Edgar H. Schein. 1979. Toward a theory of organizational socialization. Research in Organizational Behavior 1:209-264.

Mannheim, Karl. 1928/29. Das Problem der Generationen. Kölner Vierteljahreshefte für Soziologie und Sozialpsychologie 7:157-184.

Markus, Gregory B. 1979. The political environment and the dynamics of public attitudes: A panel study. American Journal of Political Science 23:338-359.

Maull, Hans W. 2006. Die prekäre Kontinuität: Deutsche Außenpolitik zwischen Pfadabhängigkeit und Anpassungsdruck. In Regieren in der Bundesrepublik Deutschland, Innen- und Außenpolitik seit 1949, Hrsg. Manfred G. Schmidt und Reimut Zohlnhöfer, 421-444. Wiesbaden: VS Verlag für Sozialwissenschaften.

Mayntz, Renate, und Hans Ulrich Derlien. 1989. Party patronage and politicization of the West German administrative elite 1970-1987 - Toward hybridization? Governance 2:384-404.

McCullagh, Peter. 1980. Regression models for ordinal data. Journal of the Royal Statistical Society, Series B (Methodological) 42:109-142.

Nolterieke, Gertruf. 1981. Beamtenkarrieren in England und Deutschland: Laufbahnstrukturen gegen Ende der sechziger Jahre. Zeitschrift für Soziologie 10:151-169.

Offe, Claus. 1970. Leistungsprinzip und industrielle Arbeit: Mechanismen der Statusverteilung in Arbeitsorganisationen der industriellen Leistungsgesellschaft. Frankfurt a.M.: Europäische Verlagsanstalt.

Overhaus, Marco, und Siegfried Schieder. 2002. Die außenpolitischen Positionen der Parteien im Bundestagswahlkampf. Politik im Netz 3(17). http://www.deutsche-aussenpolitik.de/daparchive/dateien/2002/01300.pdf. Zugegriffen: 17. Jan. 2008.

Pippke, Wolfgang. 1975. Karrieredeterminanten in der öffentlichen Verwaltung: Hierarchiebedingte Arbeitsanforderungen und Beförderungspraxis im höheren Dienst. Frankfurt a.M.: Aspekte.

Risse, Thomas. 2004. Kontinuität durch Wandel: Eine „neue“ deutsche Außenpolitik? Aus Politik und Zeitgeschichte 11:24-31.

Rouban, Luc. 2003. Politicization of the civil service. In Handbook of Public Administration, Hrsg. Guy B. Peters und Jon Pierre. London: Sage.

Rubin, Donald B. 1976. Inference and missing data. Biometrika 63:581-592.

Schnell, Rainer, Paul B. Hill, und Elke Esser. 1999. Methoden der empirischen Sozialforschung, 6. Aufl. München: Oldenbourg.

Schubert, Klaus. 2006. Leistungseliten: Die Bedeutung sozialer Herkunft als Selektionskriterium für Spitzenkarrieren - - eine Analyse unter besonderer Berücksichtigung von Sozialisation und Qualifikation. Hamburg: Kovac.

Schütz, Walter J., Hrsg. 1965. Aus der Schule der Diplomatie: Beiträge zu Außenpolitik, Recht, Kultur, Menschenführung: Festschrift für Peter H. Pfeiffer. Duisseldorf: Econ.

Schwanke, Katja, und Falk Ebinger. 2006. Politisierung und Rollenverständnis der deutschen Administrativen Elite 1970 bis 2005. Wandel trotz Kontinuität. In Politik und Verwaltung, Hrsg. Jörg Bogumil, Werner Jann, und Frank Nullmeier, 227-249. Politische Vierteljahresschrift: Sonderheft 37. Wiesbaden: VS Verlag für Sozialwissenschaft.

Sears, David O., und Carolyn L. Funk. 1991. The role of self-interest in social and political attitudes. Advances in Experimental Social Psychology 24:1-91.

Seibert, Scott E., Maria L. Kraimer, und Robert C. Liden. 2001. A social capital theory of career success. The Academy of Management Journal 44:219-237. 
Steinkemper, Bärbel. 1974. Klassische und politische Bürokraten in der Ministerialverwaltung der Bundesrepublik Deutschland: Eine Darstellung sozialstruktureller Merkmale unter dem Aspekt politischer Funktionen der Verwaltung. Köln: Heymanns.

Weigert, Manfred. 2004. Karriere im Öffentlichen Dienst, 1. Aufl. Frankfurt a.M.: Fischer.

Wenninger, Gerd. 2001. Lexikon der Psychologie. Berlin: Spektrum.

Wildenmann, Rudolf, Max Kaase, Ursula Hoffmann-Lange, Albrecht Kutteroff, und Gunter Wolf. 1982. Führungsschicht in der Bundesrepublik Deutschland 1981. Mannheim: Universität Mannheim.

Wilke, Dietrich. 1980. Aufgaben, Auswahlmodi und Ausbildungszielkonzeptionen des höheren Auswärtigen Dienstes der Bundesrepublik Deutschland. Frankfurt a.M.: Lang.

Windmeijer, Frank A. G. 1995. Goodness-of-fit measures in binary choice models. Econometric Reviews 14:101-116.

Yee, Albert S. 1997. Thick rationality and the missing „brute fact“: The limits of rationalist incorporations of norms and ideas. The Journal of Politics 59:1001-1039.

Zürn, Michael, und Jeffrey T. Checkel. 2005. Getting socialized to build bridges: Constructivism and rationalism, Europe and the nation-state. International Organization 59:1045-1079. 